\title{
Potential Impacts of Mass Nutritional Supplementation on Dynamics of Measles: A Simulation Study
}

\author{
Navideh Noori ${ }^{a,}{ }^{,}$, Laura A. Skrip ${ }^{b}$, Assaf P. Oron ${ }^{a}$, Kevin A. McCarthy, Benjamin M. Althouse ${ }^{a, c, d}$, \\ Indi Trehan ${ }^{\mathrm{e}}$, and Kevin P.Q. Phelan ${ }^{\mathrm{f}}$ \\ a Institute for Disease Modeling, Global Health Division, Bill and Melinda Gates Foundation, 5005 th Ave \\ N, Seattle, WA, 98109, USA \\ b School of Public Health, University of Liberia, 1000 Monrovia, 10 Liberia \\ C Information School, University of Washington, Seattle, WA, 98104, USA \\ d Department of Biology, New Mexico State University, Las Cruces, NM, 88003, USA \\ e Department of Global Health, University of Washington, Seattle, WA, 98104, USA

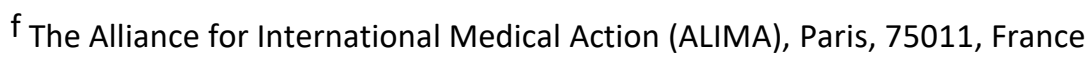 \\ * Corresponding author: Navideh Noori \\ Email: nnoori@idmod.org \\ https://orcid.org/0000-0003-3561-2720
}

Author Contributions: IT, KPQP contributed to the design and interpretation of the analysis. NN and LS developed the model. NN conducted the analysis. KAM and KMA contributed to the model revision. NN, LS, APO, KAM, BMA, IT, KPQP wrote the manuscript.

Competing Interest Statement: Kevin P.Q. Phelan, The Alliance for International Medical Action (ALIMA) serves on the Social Purposes Advisory Commission of Nutriset, a main producer of lipid-based nutrient supplement products. Other authors declare no conflict of interest.

Classification: Biological Science: Population Biology.

Keywords: wasting, measles, acute malnutrition, food supplementation, vaccination 


\begin{abstract}
The bidirectional interaction between undernutrition and infection can be devastating to child health. Nutritional deficiencies stunt growth, impair immunity, increase susceptibility to both infection and more severe outcomes from common infections. Simultaneously, infections compound undernutrition by increasing metabolic demand, reducing food intake, and impairing nutrient absorption. Treatment of acute malnutrition (wasting) can reverse at least some of its deleterious effects and reduce susceptibility to, and transmission of, infectious diseases. Nutrition-specific approaches may be packaged with other interventions, including immunization, to support overall child health. To understand how mass nutritional supplementation, treatment of wasting, and vaccination affect the dynamics of a vaccine-preventable infection, we developed a population-level, compartmental model of measles transmission of children aged 6-23 months old, stratified by nutrition status. We simulated a range of scenarios to assess the potential reductions in measles infection and mortality associated with targeted therapeutic feeding for children who are wasted and with a mass supplementation intervention. Nutrition interventions were assumed to reduce susceptibility to measles-related death and to increase engagement with the health sector, leading to increased vaccination rates. We found that if all wasted children receive therapeutic feeding, and if this leads to an increase in measles vaccination uptake, up to $12 \%, 20 \%$, and $23 \%$ reductions in measles infections, measles-attributable mortality, and overall mortality among wasted children, respectively, could be averted. Combination of wasting treatment and mass supplementation coverage followed by an increase in vaccination coverage of non-wasted children from a baseline of $75 \%$ to $85 \%$, leads to a $64-66 \%$ reduction in measles infection and mortality and $27 \%$ reduction in overall mortality among wasted children, compared with the wasting treatment alone. Our work highlights the synergistic benefits that may be achieved by leveraging mass nutritional supplementation as a touch point with the health system, to increase rates of vaccination in order to improve child survival beyond what would be expected from the additive benefits of each intervention.
\end{abstract}

\title{
Significance Statement
}

The pathways by which undernutrition and infection interact have not been fully disentangled. Undernourished children may be more susceptible to infection and at increased risk for infectious disease complications. Infection also can interfere with nutrition intake or retention. Nutrition-based interventions may have indirect benefits on reducing infectious disease burden by reducing susceptibility through multiple routes. One of these is enhancing engagement with the health sector, thereby leading to adoption of other essential services such as vaccination. Here we quantify the association between undernutrition and a vaccine-preventable disease by developing a mathematical model of measles transmission which accounts for childhood nutritional and vaccination statuses. Our results show that mass nutritional supplementation has a strong effect in reducing the burden of measles and undernutrition, compared with wasting treatment only. Our work may elevate the potential role of nutritional supplementation in infection prevention, and encourage more targeted data collection around the effectiveness of packaged care in optimizing child development and growth.

\section{Main Text}

\section{Introduction}

Undernutrition and infection interact in a bidirectional manner. Both micronutrient and macronutrient deficiencies stunt a child's growth, impair immunity, increase susceptibility to infection, and worsen outcomes from common infectious diseases (1). The risk of adverse outcomes from infectionis correlated with the degree of undernutrition; concurrently, infection contributes to undernutrition by reducing a 
child's ability to consume food, by contributing to nutrient malabsorption, and by increasing metabolic demand (2-4). As an example, a higher cumulative burden of diarrhea during the first two years of life increases the risk of stunting at 24 months of age (5). Improving the baseline nutritional status of children and providing treatment for acute malnutrition (wasting) lowers the negative impacts of infections on growth by strengthening children's immune systems, preventing poor appetite, compensating for malabsorption, and favoring the growth of beneficial gut microorganisms $(2,6)$.

Undernutrition can lead to long-lasting immune deficits, rendering undernourished children at a significantly increased risk of respiratory infections, diarrhea, malaria, and measles (3, 7-9). Even seemingly mild manifestations of these common infectious diseases can have long-term effects on children's physical and cognitive development (10). Identifying patterns of association between undernutrition and infection are important to the clinical and public health efforts in reducing childhood morbidity and mortality (1). The bidirectional relationship between the two events has been well-documented, and its importance has been recognized for decades $(4,11)$. Few studies, though, have explored the impact of undernutrition on the transmission dynamics of infectious diseases. Among these, there has been investigation into the effect of undernutrition, or specific nutritional therapies, on transmission of tuberculosis (12) and cholera (13), emphasizing the need to address nutrition to reduce the burden of the infection. There are also animal models of infection and undernutrition that show infection can cause undernutrition; specifically, weanling undernutrition exacerbates infection and mucosal disruption (14-17), and increases the intensity of infection in neonatal mice, as assessed by stool shedding, by 1-4 orders of magnitude (15).

Undernutrition is a primary contributor to death in $44.8 \%$ of childhood fatalities from measles (18). Malnourished children are more likely to develop complications of measles and have a higher case-fatality ratio (19), while measles infection can in turn worsen the nutritional status of children. Koster et al. (20) found that measles had an adverse impact on both mortality and the nutritionalstatus of surviving children. In their prospective study in Bangladesh, children 7-23 months of age experienced a persistent nutritional deficit of about $10 \%$ after measles infection followed by prolonged diarrhea. Another study in an urban settlement in Guinea-Bissau also found a negative impact of measles on the nutritional status of children aged 9-35 months old (21). Several studies have shown that measles was a precipitating cause of kwashiorkor (22-24), and that there have been reports of a reduction in the incidence of kwashiorkor following measles vaccine campaigns (23).

Interventions focused on treating or preventing undernutrition often rely upon increasing the engagement of mothers and children with the health sector. Intentionally packaging nutrition-specific care with routine immunization could lead to enhanced effectiveness in reducing undernutrition but also improve prevention efforts against infectious disease. In fact, nutritional commodities may be perceived as an incentive to draw patients to health centers where other services may be offered. Previous work has shown that an incentive as simple as a bag of lentils for each immunization visit, has large positive impacts on the uptake of immunization services in resource poor areas (25). In addition, vaccination uptake can enhance long-term nutrition outcomes, and targeted vaccination of children with poor socio-demographic characteristics can improve their overall nutrition status (26).

We developed apopulation-level, dynamic model of measles transmission stratified by nutrition status to understand how nutrition-based interventions and vaccination collectively affect measles infection and mortality. In particular, we modeled two scenarios. In the first, wasted children received treatment with ready-to-use therapeutic food (RUTF). The second included treatment of wasted children, as well as mass supplementation of all children 6-23 months with small quantity lipid-based nutritional supplements (SQLNS). Lipid-based nutritional supplements (LNS) are a class of ready-to-use food supplements that are highly nutrient-dense and fortified with vitamins and minerals at levels designed to treat and prevent acute malnutrition. They range in ration sizes based on their use to either treat or prevent acute malnutrition, with ready-to-use therapeutic or supplementary foods (RUTF, RUSF) coming in 92- or 100-gram sachets, medium-quantity LNS in 50 gram sachets, and 20-gram sachets of SQ-LNS for home fortification of local diets to improve children's complementary feeding. There is strong evidence in support of implementing mass SQ-LNS supplementation among at-risk children, like those in Niger (27). Such supplementation has been shown to improve growth and reduce the prevalence (28) and incidence (29) of wasting, and reduce 
all-cause mortality (30).

We accounted for the nature, timing, and magnitude of interactions between measles and undernutrition under the different simulated scenarios. Model parameters reflected the situation in Niger, which was considered to be an appropriate case study due to its high rates of undernutrition and its persistent measles burden. Undernutrition is the leading cause of infant mortality in Niger (31). The levels of undernutrition in Niger are among the highest in the world, with $48.5 \%$ and $14.1 \%$ of children under 5 years of age being stunted and wasted in 2018, respectively (32). These numbers are higher than the average for the African continent as a whole $(29.1 \%$ and $6.4 \%$ respectively). Outbreaks of vaccinepreventable diseases like measles are also common in Niger (33).

Our modeling scenarios can help us understand the complex connections between undernutrition and measles that have not been disentangled by previous studies and may help inform approaches to intervention. There is strong evidence for the positive impact on preventing undernutrition and mortality through mass supplementation with child-adapted foods like SQ-LNS. If our model demonstrates an additive benefit of increased measles vaccination, it would justify further investment by governments and donors in scalingup such programs especially in areas with a simultaneous high burden of malnutrition and low coverage for measles vaccination.

\section{Results}

Scenario 1. We modeled a 20\% prevalence of wasting among children aged 6-23 months. Treating wasted children was assumed to increase vaccination coverage among wasted children seeking treatment. We first assumed a coverage of therapeutic treatment for wasted children to be $20 \%$ with $75 \%$ effectiveness and explored the impact of incremental increases, along with constant or increased vaccination coverage among the treated children only, upon measles infection, measles mortality and overall mortality. An underlying assumption is that unvaccinated wasted children who receive therapeutic feeding are given the measles vaccine during week 4 of treatment, as is indicated by most treatment protocols. Fig. 2 shows how the combination of wasting treatment and measles vaccination coverage impacts measles outcomes. For a fixed vaccination coverage of $67.5 \%$ among wasted children, treatment of wasting alone could lead to up to a $6 \%$ reduction in measles cases, and $17 \%$ reduction in measles mortality, when coverage levels of treatment were $20-100 \%$. In addition to these benefits seen for the overall population of children, the combination of wasting treatment coverage from $20 \%$ to $100 \%$ followed by measles immunization coverage from $67.5 \%$ to $100 \%$ reduces the measles infection and mortality among wasted children specifically by $26 \%$, and overall mortality of wasted children by $23 \%$ (Fig. S3). Varying the baseline treatment coverage of wasted children (20\%, 30\% and $40 \%)$ and baseline vaccination coverage of children treated for wasting $(65 \%, 70 \%, 75 \%$, $80 \%$ ) reduce measles burden in a non-linear manner (Fig. S4 and Fig. S5). Higher baseline coverages reduce number of measles cases and deaths, but also reduces the effect size.

Scenario 2. Instead of therapeutic feeding of wasted children, we included mass supplementation for nutritionally at-risk populations - a less-targeted intervention with greater reach at the community-level. We assumed that $60 \%$ of the children 6-23 months old received mass supplementation with SQ-LNS at health centers and hypothesized that vaccination coverage of non-wasted children $\left(S_{N 0}\right)$ who received supplementation increased from $75 \%$ to $85 \%$. This led to a $19 \%$ and $9 \%$ reduction in measles infection and mortality, respectively.

Scenario 3. We combined Scenarios 1 and 2 and assessed the effects of therapeutic feeding of wasted children and mass supplementation of $60 \%$ of children 6-23 months old, together on measles outcomes. We varied the treatment coverage of wasted children $(\tau)$ between $20 \%$ to $100 \%$ as well as the vaccination coverage of treated children for wasting (ut) between $67.5 \%$ to $100 \%$. An increase in vaccination coverage of non-wasted children as a result of mass supplementation $\left(u^{\prime}\right)$ from $75 \%$ to $85 \%$, leads to a $19-20 \%$ reduction in measles infection and $9-11 \%$ reduction in measles mortality (Fig. 3). Varying the baseline treatment coverage of wasted children does not change the effect size noticeably (Fig. S6).

Comparing Scenario 1 with Scenario 3 reveals that the absolute numbers of measles cases and measles mortality are much lower in Scenario 3 than Scenario $1.85 \%$ vaccination coverage of non-wasted children 
following mass supplementation, in addition to varying wasting treatment and vaccination coverage of children treated for wasting, leads to $65-66 \%$ and $64-65 \%$ reductions in measles infection and mortality, respectively, compared with wasting treatment only (Fig. 4a and Fig. S7). Among wasted children specifically, the infection and mortality due to measles also reduced by $64-65 \%$, and overall mortality by $27 \%$, for the $85 \%$ vaccination coverage (Fig. S8).

Effects of Scenario 3 and Scenario 2 on model outcomes are similar. If mass supplementation leads to $85 \%$ vaccination coverage of non-wasted children in both Scenarios 2 and 3, comparing Scenario 3 outcomes with Scenario 2 shows similar amount of reduction in model outcomes (Fig. 4b). Increasing the baseline treatment coverage of wasted children increases the effect size of Scenario 3 in comparison to Scenario 2 and leads to larger reduction in measles infection, measles mortality and overall mortality (Fig. S9 and Fig. S10).

These differences in model outcomes were estimated by fixing the input parameter values to best estimates informed by the literature (Table 1). However, precise quantities to inform some of the model parameters are unknown or unavailable, specifically, the increase in infectious period and increase in susceptibility to measles due to wasting ( $\eta$ and $\theta$ ). Simulation over a range of values for one parameter with others fixed allows us to examine the effect of Scenario 3 compared with Scenario 1 on multiple model outcomes. As the magnitude of wasting effects - such as susceptibility effect and duration of infectious period - get larger, they result in an increase in the number of infected children, and number of deaths, and also an increase in the effect size of mass supplementation and wasting treatment, compared with wasting treatment only (Fig. S11). For instance, an increase in susceptibility effect from $\theta=2$ to $\theta=4$, leads to a $61 \%-65 \%$ in measles infection (Fig. S11). Varying other parameter values does not lead to a noticeable difference in the simulation results.

Further sensitivity analyses using the LHS-partial rank correlation coefficient (LHS-PRCC) index and generating a matrix of 5000 sample points in the 9-dimensional unit cube (all parameters) indicate that $\eta$ (Infectious period extension for wasted children) and $\theta$ (susceptibility impact of measles infection) have the strongest influence on both measles infection and measles mortality outcomes (Fig. S12). In addition, mortality due to measles is sensitive to $\sigma$ (measles-induced mortality probability) and $\phi$ (increased measles mortality due to wasting).

\section{Discussion}

Substantive barriers to improving child health in resource-limited settings include persistently high rates of undernutrition and low rates of vaccine coverage for common infections such as measles. Given the close bidirectional relationship between childhood undernutrition and infectious diseases among vulnerable populations, there may be opportunities for synergy wherein the treatment and/or prevention of one condition leads to decreased rates of the other. Our work aims to capture the dynamic interactions between undernutrition and measles, so as to estimate the impact of treatment of wasting, mass nutritional supplementation for nutritionally at-risk populations, and measles vaccine coverage on measles infection and mortality.

Our models demonstrate a strong effect of mass nutritional supplementation in reducing measles infection and death, as well as overall mortality. Importantly, modestly increasing measles vaccination coverage by providing vaccination at the same time and location as blanket nutritional supplementation leads to sizeable reductions in measles cases and mortality well above treatment of wasting or increased vaccine uptake alone.

Our results show that mass supplementation alone and mass supplementation combined with wasting treatment have a similar effect size on reducing measles infection and mortality. Mass supplementation with SQ-LNS coupled with vaccination would likely be more cost-effective than the individual treatment of wasting alone which, in high burden countries, puts financial pressures on vulnerable and poorly resourced health systems. Future work should explore the cost-effectiveness of integrated interventions.

We used model parameters based on data from Niger, a nation plagued by a high burden of 
undernutrition, where interventions focused on the early childhood nutrition have the potential for a large impact on measles infection rates. Niger has made very little progress towards achieving international targets for decreasing stunting and wasting (34). Our results suggest that mass nutritional supplementation among vulnerable populations using SQ-LNS has over twice the effect on reducing measles infection and mortality as well as overall mortality among wasted children, compared with treatment of wasted children alone, assuming both methods lead to increased vaccination coverage. Therefore, expanding the reach of mass nutritional supplementation, including providing nutrition supplementation during routine immunization visits, could help Niger make progress towards lowering the incidence of wasting, as well as indirectly improve vaccination coverage. The models could be applied to other countries like Chad, Nigeria, or the Democratic Republic of Congo which have a similar burden of undernutrition as Niger but much lower measles vaccination coverage.

As with all models, there are some limitations to our work. Although we account for multiple interactions between measles and undernutrition, there are several features of the complex system of measles epidemiology that were not included in the model. For instance, we did not account for any potential impact of undernutrition on measles vaccine leading to a potential delay in response to vaccine or waning of vaccine, as it is under-explored in the literature (35). Also, our model does not account for older children and adults, which would play a role in measles infections between age groups. In addition, one of the complications that malnourished children experience following measles infection, is post-measles diarrhea which exacerbates nutrient loss (1). Accounting for this could help us better identify the impacts of infection on children's nutritional status. Also, among surviving children following measles infection a certain percentage experience blindness, a certain percentage with some residual pneumonia, and quite significantly a certain percentage with a residual post-measles immunodeficiency. The reported deaths due to measles do not account for mortality from these various comorbidities (36-37). In addition, assessing the potential increased pathogenicity of measles as a result of undernutrition would be useful in understanding the cyclic relationship between the two events to develop more effective interventions. Considering that our model outputs are sensitive to some of the model parameters, more field data to constrain parameters around the relationship between measles infection and undernutrition would allow for increasingly rigorous quantification of direct and indirect impacts of nutrition interventions. Also, nutrition interventions that led to rises in vaccination coverage were assumed to be delivered at health centers and at regular intervals. It will also be important to investigate different distribution models for supplementation in order to retain contact with health personnel without placing undue burden on already overworked caretakers.

Given the complex interactions between undernutrition and infectious diseases, it is still challenging to recognize where and at what level specific interventions can be most effective. The large contribution of undernutrition and infection to the global burden of disease increases the importance of studying these two processes. Our study offers a more nuanced understanding of the complex interactions between measles transmission dynamics and undernutrition and helps motivate advocacy for nutrition-focused interventions based on their multidimensional impacts. This work also helps understand how the addition of nutritional status, preventive nutrition interventions, and therapeutic treatments into mathematical models of vaccine-preventable diseases can better explain increased susceptibility to infection despite achieving certain vaccine coverage levels. This model also has implications for understanding other indirect benefits of increased care-seeking due to engagement with the health system for nutrition-based interventions. When considering how health systems with limited budgets should spend their money to achieve maximal benefit, we have shown here that leveraging mass nutritional supplementation as a contact point with the health system to even modestly increase measles vaccination coverage has a synergistic benefit beyond either intervention alone.

\section{Materials and Methods}

Measles-Undernutrition Model with Wasting Treatment (Scenario 1). We developed a seasonally-forced, deterministic, continuous-time Susceptible-Infectious-Recovered (SIR) model of measles transmission that 
accounts for vaccination and for nutritional interventions (Fig. 1). We stratified the population into children 6-23 months and individuals of all other ages, with children aging from the former into the latter compartment at a rate $\alpha$. We assumed a fixed population of $N_{0}=100,000$ and the demographic characteristics of Niger such as birth rate and death rates (38-40). The population size of infants up to 6 months is assumed to be $B=5000$. A full list of parameter values is given in Table 1 , and model equations are also given in supplementary material.

Initially, children enter the two classes of susceptibility based on their nutritional status: nourished (nonwasted, $\left.S_{N O}\right)$ or wasted $\left(S_{M O}\right)$. A proportion, $\omega$, of children become wasted during the first 6 months of life. Some of the wasted children receive therapeutic feeding and move from $S_{M O}$ to the nourished and treated compartment $\left(S_{N_{1}}\right)$. The baseline treatment coverage of wasted children is assumed to be $20 \%$ with $75 \%$ effectiveness at a rate $\tau=0.75 * 0.2 / 365$ day. Previous studies have shown that after recovery from wasting, some children relapse and become wasted post-discharge (41). We accounted for relapse by moving treated individuals from $\left(S_{N 1}\right)$ compartment to $\left(S_{M 1+}\right)$ at rate $\chi$. After individuals in $S_{M 1+}$ are treated again for wasting, they move to $\left(S_{N_{2}}\right)$ and can in turn, move back to $\left(S_{M 1+}\right)$ after experiencing relapse for the second time or more. We assume that wasted children have a higher mortality rate $(\mu m)$ than in other states.

A proportion of individuals from all above-mentioned compartments are vaccinated and enter the vaccinated compartment $(\mathrm{V})$. The vaccination coverage is assumed to be $75 \%$, close to Niger national immunization coverage in 2016 (42). We model single-dose vaccination and assume $95 \%$ of vaccinated children are no longer susceptible to measles infection. We also assumed that vaccination coverage of wasted children are $10 \%$ lower than nourished children (uw $=67.5 \%)$, based on the Demographic and Health Surveys data (43). Based on observations of changes in overall care-seeking as a result of engagement of mothers through nutrition intervention programs (25), we hypothesize that receiving therapeutic feeding for wasting increases the measles vaccine uptake by $v t$.

Upon contracting measles, nourished and wasted individuals enter the infectious compartments $\left(I_{N}\right.$ and $I_{M}$, respectively). Depending on the host age and condition, measles may be fatal. This is presented by the measles case fatality rate, here defined as probability $(\sigma)$. We assume that wasting leads to increased susceptibility to measles $(\theta)$, longer infectious period $(\eta)$, and increased measles mortality $(\phi)$. In this work, we assumed that the subsequent wasting event increases susceptibility to measles to the same degree as the first wasting event $(\varsigma=0)$. Individuals who recover from measles move to the recovered class $(R)$. A proportion, $H$, of children develop wasting after measles infection (23). Children who survive from measles but become wasted post-measles infection move from $I_{N}$ to $R_{N M}$, and those who stay nourished move to $R_{N N}$ class. There is also a chance, $\rho$, of mortality due to measles-associated wasting $(24,44)$. These parameter values were informed by literature on kwashiorkor specifically, due to a lack of studies on measlesassociated marasmus. We assume that a proportion, $\delta$, of wasted children who get infected with measles, eventually recover from wasting and move from $I_{M}$ to $R_{M N}$ upon infection. Those who stayed wasted, move from $I_{M}$ to $R_{M M}$.

We depict the findings as heatmaps displaying measles infections averted across different combinations of therapeutic feeding coverage for children with wasting $(\tau)$ and coverage of vaccination among wasted children receiving therapeutic feeding $(u t)$. We vary these two quantities while fixing values of the remaining model parameters. The percentage reduction in infections and mortality due to measles among all children aged 6-23 months old, infections and mortality due to measles among wasted children, and overall mortality among wasted children are the model outcomes. To calculate the percentage reduction, the sum of number of each outcome over the last year of simulations were calculated versus the baseline $\operatorname{sum}(\tau=0.2$ and $u t=67.5)$.

Measles-Undernutrition Model with Mass Nutritional Supplementation (Scenario 2). Instead of wasting treatment, we model the effect of community-wide mass nutritional supplementation on dynamics of measles by assuming that $60 \%$ of the population aged 6-23 months (parameter $M C$ ) receive daily doses of SQ-LNS (20 gr per sachet) at health center, per coverage found in a recent study in Mali (29, Table 3) (Fig. 
S1). We hypothesize that the mass supplementation results in an increase in the first dose of measles vaccine uptake in the region during the time the cohort receives supplementation. We represent this effect by conducting four scenarios and changing measles vaccination coverage from the baseline value of $75 \%$ to $80 \%$, and $85 \%$ among susceptible individuals who are nourished $\left(S_{N 0}\right)$ after receiving the SQ-LNS. The increased measles vaccine uptake is shown as $U^{\prime}$ (Fig. S1). Recent studies have shown significant improvement in children's nutritional status as a result of receiving SQ-LNS. A cluster-randomized controlled trial in Mali has shown a $29 \%$ reduction in the probability of a first wasting event (29). We account for this effect in the model by parameter $k$ (Fig. S1). Also, a recent pooled analysis of randomized controlled trials of LNS showed a $27 \%$ reduced probability of all-cause mortality (30). This effect is shown as $\mu m^{\prime}$ in the model.

The number of measles cases is calculated as the sum of (1) the number of measles cases where $40 \%$ of the population (1-MC) do not receive the mass supplementation, and (2) the number of measles case where $60 \%$ of population $(M C)$ receive the mass supplementation, per unit time. The number of measles cases among wasted children, mortality due to measles among wasted children, and overall mortality among wasted children were calculated in a similar manner.

Measles-Undernutrition Model with Mass Nutritional Supplementation and Wasting Treatment (Scenario 3). We combine scenarios 1 and 2 and we model the combined effects of wasting treatment and the community-wide mass nutritional supplementation on dynamics of measles (Fig. S2).

Sensitivity Analysis. To characterize the response of model outputs to parameter variations and to identify the influential parameters, we conducted a sensitivity analysis using Latin hypercube sampling-partial rank correlation coefficient (LHS-PRCC). LHS-PRCC is an efficient sampling-based sensitivity analysis that assumes the effect of a parameter on model outputs is monotonic after removing the linear effects of all parameters except the parameter of interest (45). Using LHS, we generated a matrix of 5000 sample points in the 9dimensional unit cube (i.e., all parameters), and we explored how these parameters affected the model outcomes by using different simulated parameter sets. Next, we determined the effect of each parameter on the model outcomes while averaging over the other variables by inspecting the PRCC between each of the parameters and the percent change in different outcomes.

\section{Acknowledgments}

The funders had no role in study design, data collection, data analysis, the decision to publish, or preparation of the manuscript. The content of this article is solely the responsibility of the authors and does not necessarily represent the official views of their respective employers or funders.

\section{References}

1. D.L. Humphries, M.E. Scott, S.H. Vermund, "Nutrition and infectious diseases, shifting the clinical paradigm" (Springer Nature Switzerland AG 2021).

2. N.S. Scrimshaw, Historical concepts of interactions, synergism and antagonism between nutrition and infection. J. Nutr. 133, 316-321 (2003).

3. U.E. Schaible, S.H. Kaufmann, Malnutrition and infection: Complex mechanisms and global impacts. PLoS Med. 4, 0806-0812 (2007).

4. J.L. Walson, J.A. Berkley, The impact of malnutrition on childhood infections. Curr. Opin. Infect. Dis. 31, 231-236 (2018).

5. W. Checkley et al., Multi-country analysis of the effects of diarrhoea on childhood stunting. Int. J. Epidemiol. 37, 816-830 (2008).

6. K.G. Dewey, D.R. Mayers, Early child growth: How do nutrition and infection interact? Matern. 
Child Nutr. 7, 129-142 (2011).

7. R.E. Black et al., Maternal and child undernutrition: global and regional exposures and health consequences. The Lancet. 371, 243-260 (2008).

8. P. Katona, J. Katona-Apte, The interaction between nutrition and infection. Clin. Infect. Dis. 46, 1582-1588 (2008).

9. Z.A. Bhutta et al., Severe childhood malnutrition. Nat. reviews. Dis. Primers. 3, 17067 (2017).

10. R.E. Black et al., Maternal and child undernutrition and overweight in low-income and middleincome countries. The Lancet. 382, 427-451 (2013).

11. C.D. Bourke, J.A. Berkley, A.J. Prendergast, Immune dysfunction as a cause and consequence of malnutrition. Trends Immunol. 37, 386-398 (2016).

12. O. Oxlade, Ch.-C. Huang, M. Murray, Estimating the impact of reducing under-nutrition on the tuberculosis epidemic in the central eastern states of India: A dynamic modeling study. PLoS One. 8, 1-15(2015).

13. S.D. Hove-Musekwa et al., Modelling and analysis of the effects of malnutrition in the spread of cholera. Math. Comput. Model. 53, 1583-1595 (2011).

14. B.P. Coutinho et al., Cryptosporidium infection causes undernutrition and, conversely, weanling undernutrition intensifies infection. J. Parasitol. 94, 1225-1232 (2008).

15. J.K. Roche, A. Cabel, J. Sevilleja, J. Nataro, R.L. Guerrant, Enteroaggregative Escherichia coli (EAEC) impairs growth while malnutrition worsens EAEC infection: A novel murine model of the infection malnutrition cycle. J. Infect. Dis. 202, 506-514 (2010).

16. L.B. Costa et al., Novel in vitro and in vivo models and potential new therapeutics to break the vicious cycle of cryptosporidium infection and malnutrition. J. Infect. Dis. 205, 1464-1471 (2012).

17. L.A. Bartelt et al., Persistent G. lamblia impairs growth in a murine malnutrition model. J. Clin. Invest. 123, 2672-2684(2013).

18. M.K. Ibrahim, M. Zambruni, C.L. Melby, P.C. Melby, Impact of childhood malnutrition on host defense and infection. Clin. Microbiol. Rev. 30, 919-971 (2017).

19. B. Fenn, Malnutrition in Humanitarian Emergencies. Midwifery. 4 (2014).

20. F.T. Koster, G.C. Curlin, K.N. Aziz, A. Haque, Synergistic impact of measles and diarrhoea on nutrition and mortality in Bangladesh. Bull. World Heal. Organ. 59, 901-908 (1981).

21. L. Smedman, A. Lindeberg, O. Jeppsson, R. Zetterstrom, Nutritional status and measles: A community study in Guinea-Bissau. Annals Trop. Paediatr. 3, 169-176 (1983).

22. D. Morley, Severe measles in the tropics. Br. Med. J. 1, 297-300 (1969).

23. D.C. Morley, Measles in the developing world. Proc. Royal Soc. Med. 67, 1112-1115 (1974).

24. N.S. Scrimshaw, J.B. Salomon, H.A. Bruch, J.E. Gordon, Studies of diarrheal disease in central America VIII. measles, diarrhea, and nutritional deficiency in rural Guatemala. Am. J. Trop. Med. Hyg. 15, 625-631 (1966).

25. A.V. Banerjee, E. Duflo, R. Glennerster, D. Kothari, Improving immunisation coverage in rural India: Clustered randomised controlled evaluation of immunisation campaigns with and without incentives. BMJ. 340, 1291 (2010).

26. R. Sato, Association between uptake of selected vaccines and undernutrition among Nigerian children. Hum. Vaccin. Immunother. 1-9 (2021). DOI: 10.1080/21645515.2021.1880860

27. E.C. Keats et al., Effective interventions to address maternal and child malnutrition: an update of the evidence. Lancet. Child. Adolesc. Health. 5, 367-384 (2021).

28. J.K. Das et al., Preventive lipid-based nutrient supplements given with complementary foods to infants and young children 6 to 23 months of age for health, nutrition, and developmental outcomes. Cochrane Database of Systematic Reviews. 5 (2019). DOI: 10.1002/14651858.CD012611.pub3.

29. L. Huybregts et al., Impact on child acute malnutrition of integrating small-quantity lipid-based nutrient supplements into community-level screening for acute malnutrition: A clusterrandomized controlled trial in Mali. PLoS Med. 16, 1-31 (2019). 
30. C.P. Stewart et al., Lipid-based nutrient supplements and all-cause mortality in children 6-24 months of age: A meta-analysis of randomized controlled trials. Am. J. Clin. Nutr. 111, 207218 (2020).

31. IF of Red Cross, RC Societies, Case study Tackling malnutrition in Niger. (2020).

32. UNICEF/WHO/World Bank Joint Child Malnutrition Estimates Expanded Database, Stunting, Wasting and Overweight (2020).

33. World Health Organization Regional Office for Africa, Weekly bulletin on outbreaks and other emergencies, Week 07: 08 - 14 February 2021, (World Health Organization), Technical Report February (2021).

34. A. Osgood-Zimmerman et al., Mapping child growth failure in Africa between 2000 and 2015. Nature 555, 41-47 (2018).

35. A.J. Prendergast, Malnutrition and vaccination in developing countries. Philos. Trans. R. Soc. Lond. B. Biol. Sci. 370 (2015).

36. B.M. Laksono et al., Studies into the mechanism of measles-associated immune suppression during a measles outbreak in the Netherlands. Nat. Commun. 9, 4944 (2018).

37. M.J. Mina, C.J.E. Metcalf, R.L. de Swart, A.D.M.E. Osterhaus, B.T. Grenfell, Long-term measlesinduced immunomodulation increase overall childhood infectious disease mortality. Science. 348(6235):694-9 (2015).

38. M. Oumarou, M.I. Kanta, G. Le Duc, The new role of Nigerian medical NGOs in treating SAM. (2011).

39. World Bank World Development Indicators, Birth rate, crude (per 1,000 people) - Niger Data (2016).

40. World Bank World Development Indicators, Mortality rate, infant (per 1,000 live births) - Niger Data (2016).

41. H.C. Stobaugh et al., Relapse after severe acute malnutrition: A systematic literature review and secondary data analysis. Matern. Child Nutr. 15, 1-12 (2019).

42. WHO, WHO vaccine-preventable diseases: monitoring system (2020).

43. DHS, The DHS Program - Quality information to plan, monitor and improve population, health, and nutrition programs (2018).

44. D. Morley, M. Woodland, W.J. Martin, Measles in Nigerian children. J. Hyg. 61, 115-134 (1963).

45. J. Wu, R. Dhingra, M. Gambhir, J.V. Remais, Sensitivity analysis of infectious disease models: methods, advances and their application. J. R. Soc. Interface. 10 (2013).

46. M. Kerac, S. Frison, N. Connell, B. Page, K.M. McGrath, Informing the management of acute malnutrition in infants aged under 6 months (MAMI): Risk factor analysis using nationallyrepresentative demographic health survey secondary data. PeerJ (2019). doi: $10.7717 /$ peerj.5848

47. S. Isanaka et al., Effect of preventive supplementation with ready-to-use- therapeutic food on the nutritional status, mortality and morbidity of children 6 to 60 months in Niger: a cluster randomized trial. JAMA 301, 277-285 (2009).

48. D, Besada et al., Niger's child survival success, contributing factors and challenges to sustainability: A retrospective analysis. PLOS ONE 11, 1-18 (2016).

49. F.M. Guerra et al., The basic reproduction number (R0) of measles: a systematic review. The Lancet Infect. Dis. 17, e420-e428 (2017).

50. M.J. Ferrari et al., The dynamics of measles in sub-Saharan Africa. Nature 451, 679-684 (2008).

51. M.J. Ferrari et al., Rural-urban gradient in seasonal forcing of measles transmission in Niger. Proc. Royal Soc. B: Biol. Sci. 277, 2775-2782 (2010).

52. A, Portnoy et al., Estimates of case-fatality ratios of measles in low-income and middle-income countries: A systematic review and modelling analysis. The Lancet Glob. Health. 7, e472-e481 (2019).

53. J. Dossetor, H.C. Whittle, B.M. Greenwood, Persistent measles infection in malnourished children. Br. Med. J. 1, 1633-1635 (1977). 
medRxiv preprint doi: https://doi.org/10.1101/2021.09.10.21263402; this version posted September 15, 2021. The copyright holder for this preprint (which was not certified by peer review) is the author/funder, who has granted medRxiv a license to display the preprint in perpetuity.

It is made available under a CC-BY 4.0 International license .

\section{Figure Legends}

Figure 1. Schematic of measles-undernutrition model with no mass nutritional supplementation (Scenario 1).

Figure 2. Combined impact of treatment of wasted children and their vaccination coverage (Scenario 1) on reduction in a) measles cases and b) mortality due to measles.

Figure 3. Impacts of mass nutritional supplementation and wasting treatment (scenario 3), on reducing measles infection and mortality due to measles among children aged 6-23 months old, assuming the vaccination coverage of nourished children $\left(u^{\prime}\right)$ after receiving the SQ-LNS changes from its baseline value of $75 \%$ to $80 \%$, and $85 \%$. The treatment coverage of wasted children $(\tau)$ was varied between $20 \%$ to $100 \%$, and the vaccination coverage of wasted children was varied between $67.5 \%$ to $100 \%$.

Figure 4. Difference between a) wasting treatment (Scenario 1) and mass nutritional supplementation and wasting treatment (Scenario 3) and b) mass nutritional supplementation (Scenario 2) and scenario 3 , in reducing measles infection (blue) and mortality due to measles (purple) among children aged 623 months old, assuming the vaccination coverage of nourished children $\left(u^{\prime}\right)$ after receiving SQ-LNS varies to $75 \%, 80 \%$, and $85 \%$. The treatment coverage of wasted children $(\tau)$ was varied between $20 \%$ to $100 \%$, and the vaccination coverage of wasted children was varied between $67.5 \%$ to $100 \%$. 
Table 1. Parameter values used in the dynamical measles-undernutrition model.

\begin{tabular}{|c|c|c|c|}
\hline Parameters & Symbol & Value & Reference \\
\hline \multicolumn{4}{|l|}{ Demographic and undernutrition parameters } \\
\hline Population size & No & 100,000 & - \\
\hline Population of surviving infants under- 6 months old & B & 5,000 & - \\
\hline Aging rate of age group 6-23 months & $\alpha$ & $1 /(6+23) / 2 \sim 1 / 15$ month $^{-1}$ & - \\
\hline Under-2 mortality rate & $\mu$ & 2.05 deaths per 10,000 per day $\sim 0.075$ per year & (36) \\
\hline Proportion of infants under- 6 months who are wasted & $\omega$ & $20 \%$ & (46) \\
\hline Wasting mortality rate & $\mu m$ & $0.016+0.075$ & $(36,47)$ \\
\hline Wasting treatment coverage & $\tau$ & variable (baseline: $20 \%, 30 \%, 40 \%$ ) 0.2/365 day & $(48)$ \\
\hline Relapse rate & $\chi$ & $2 \%$ at 1 month post-discharge & (39) \\
\hline \multicolumn{4}{|l|}{ Measles parameters } \\
\hline Measles basic reproductive ratio & Ro & 15 & (49) \\
\hline Seasonality amplitude of measles & $b_{1}$ & 0.6 & (50) \\
\hline Force of infection & $\lambda$ & See Supplementary Material & - \\
\hline Measles infectious period & $1 / \gamma$ & 14 days & $(50)$ \\
\hline Immigration rate of measles & $\epsilon$ & 10 infected per year & (51) \\
\hline Vaccination coverage of measles & $u$ & $75 \%$ & $(42)$ \\
\hline Vaccination coverage of wasted children & uw & $67.5 \%$ & (43) \\
\hline Vaccination coverage of children treated for wasting & $u t$ & variable & - \\
\hline Measles case fatality rate & $\sigma$ & $2.8 \%$ & $(52)$ \\
\hline Increased measles mortality due to wasting & $\phi$ & $(6+3.7) / 2 \sim 5$ & (7) \\
\hline Increased susceptibility to measles infection due to wasting & $\theta$ & 2 & Assumed \\
\hline Proportion of children who become wasted after measles infection & $\mathrm{H}$ & $4 \%$ & $(24,44)$ \\
\hline $\begin{array}{l}\text { Increased susceptibility to measles due to subsequent wasting event } \\
\text { Measles-associated wasting mortality }\end{array}$ & 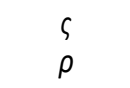 & $\begin{array}{c}0 \\
44 \% \\
\end{array}$ & $\begin{array}{l}\text { Assumed } \\
(44)\end{array}$ \\
\hline Infectious period extension for wasted children* & $\eta$ & $\begin{array}{l}\text { Assuming infectious period among wasted children } \\
\text { is } 20 \text { days, } \eta=14 / 20 \sim 0.7\end{array}$ & $(53)$ \\
\hline \multicolumn{4}{|l|}{ Mass nutritional supplementation parameters } \\
\hline Mass supplementation coverage & $M C$ & $60 \%$ & Assumed \\
\hline Measles vaccine coverage after mass supplementation & $u^{\prime}$ & $75 \%, 80 \%, 85 \%$ & Assumed \\
\hline Reduced all-cause mortality due to mass supplementation & $\mu m^{\prime}$ & $0.075(1-0.27)+0.016$ & (30) \\
\hline Reduced probability of first AM event due to mass supplementation & $K$ & $29 \%$ & (29) \\
\hline
\end{tabular}

\footnotetext{
${ }^{*}$ Measles latent and infectious periods are approximately 6 and 8 days, respectively. Infectiousness starts about 4 days before the onset of rash and lasts $\sim 4$ days after the onset of rash.
} 
medRxiv preprint doi: https://doi.org/10.1101/2021.09.10.21263402; this version posted September 15, 2021. The copyright holder for this preprint (which was not certified by peer review) is the author/funder, who has granted medRxiv a license to display the preprint in perpetuity.

It is made available under a CC-BY 4.0 International license .

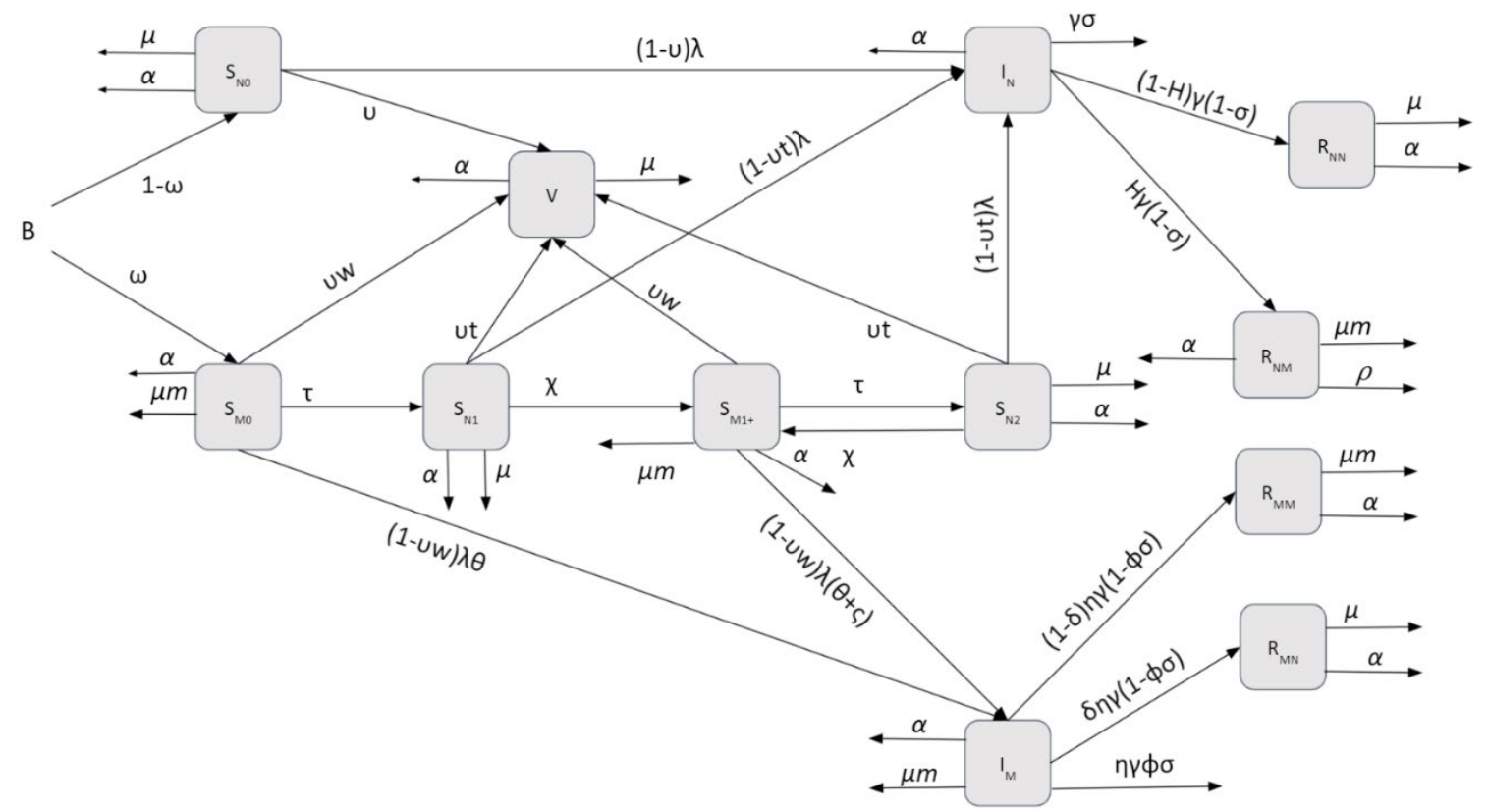

Figure 1. Schematic of measles-undernutrition model with no mass nutritional supplementation (Scenario 1). 
medRxiv preprint doi: https://doi.org/10.1101/2021.09.10.21263402; this version posted September 15, 2021. The copyright holder for this preprint (which was not certified by peer review) is the author/funder, who has granted medRxiv a license to display the preprint in perpetuity.

It is made available under a CC-BY 4.0 International license .

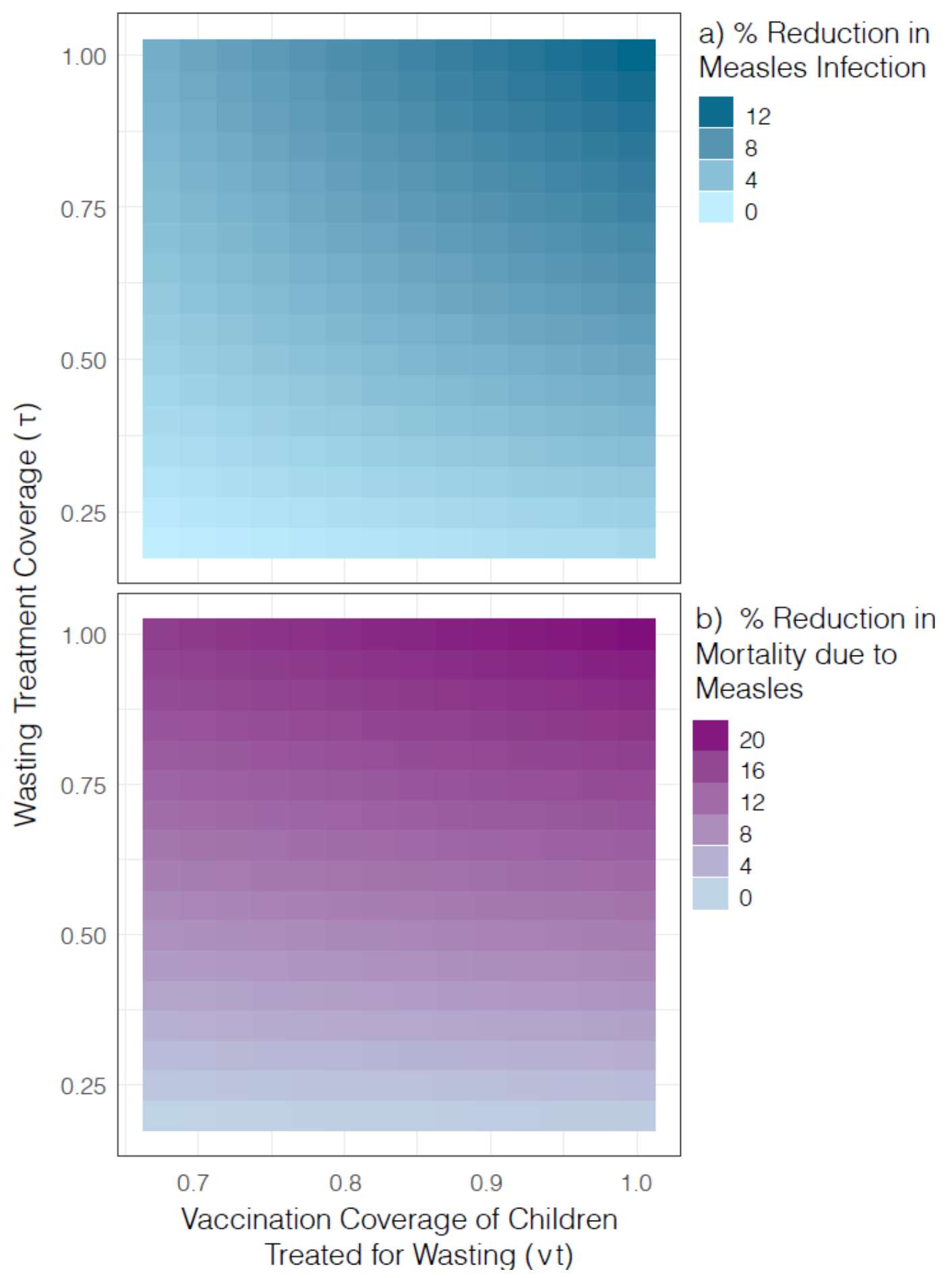

Figure 2. Combined impact of treatment of wasted children and their vaccination coverage (Scenario 1 ) on reduction in a) measles cases and b) mortality due to measles. 
medRxiv preprint doi: https://doi.org/10.1101/2021.09.10.21263402; this version posted September 15, 2021. The copyright holder for this preprint (which was not certified by peer review) is the author/funder, who has granted medRxiv a license to display the preprint in perpetuity.

It is made available under a CC-BY 4.0 International license .

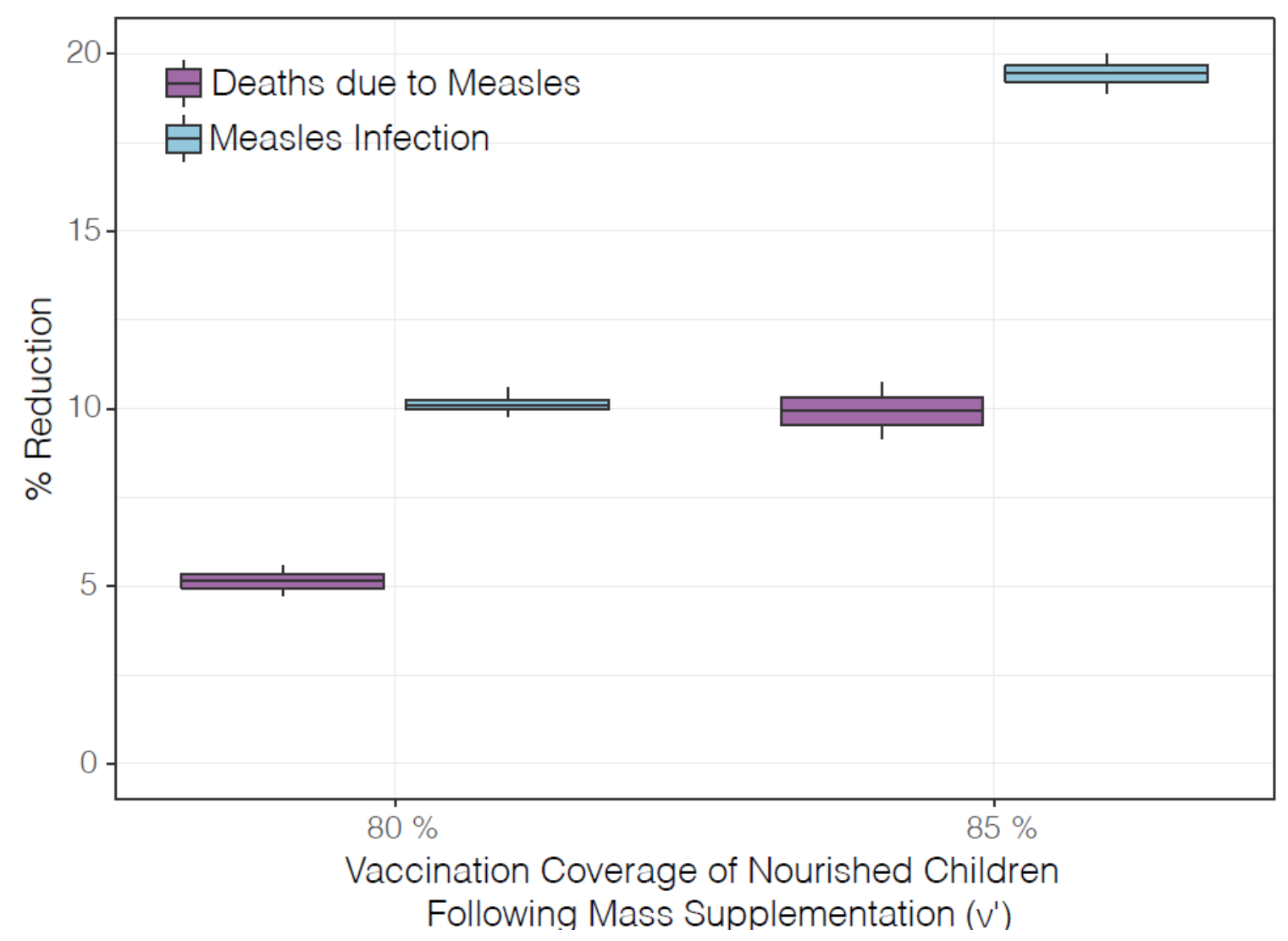

Figure 3. Impacts of mass nutritional supplementation and wasting treatment (scenario 3 ), on reducing measles infection and mortality due to measles among children aged 6-23 months old, assuming the vaccination coverage of nourished children $\left(u^{\prime}\right)$ after receiving the SQ-LNS changes from its baseline value of $75 \%$ to $80 \%$, and $85 \%$. The treatment coverage of wasted children $(\tau)$ was varied between $20 \%$ to $100 \%$, and the vaccination coverage of wasted children was varied between $67.5 \%$ to $100 \%$. 
medRxiv preprint doi: https://doi.org/10.1101/2021.09.10.21263402; this version posted September 15, 2021. The copyright holder for this preprint (which was not certified by peer review) is the author/funder, who has granted medRxiv a license to display the preprint in perpetuity.

It is made available under a CC-BY 4.0 International license .

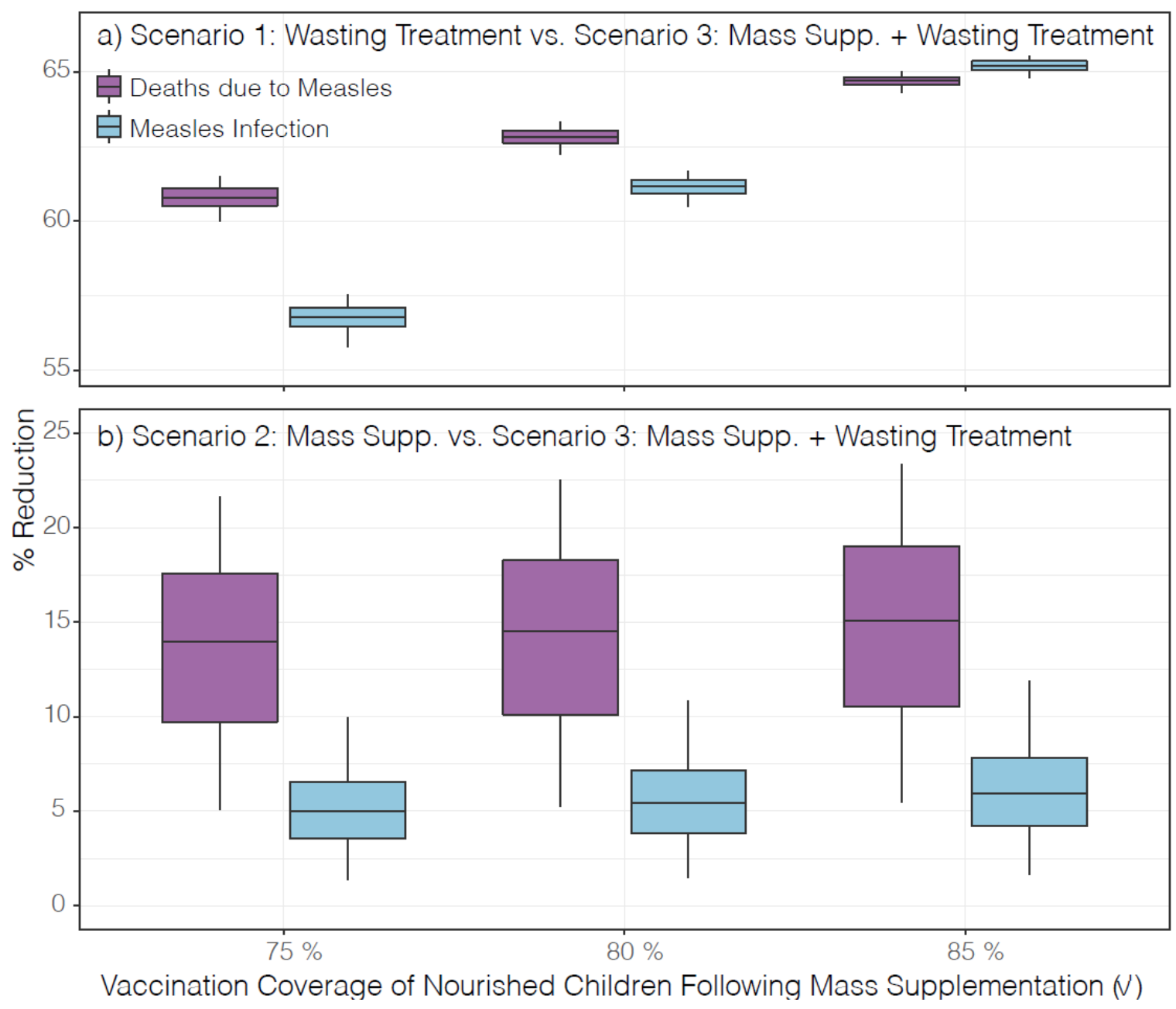

Figure 4. Difference between a) wasting treatment (Scenario 1) and mass nutritional supplementation and wasting treatment (Scenario 3) and b) mass nutritional supplementation (Scenario 2) and scenario 3 , in reducing measles infection (blue) and mortality due to measles (purple) among children aged 623 months old, assuming the vaccination coverage of nourished children $\left(u^{\prime}\right)$ after receiving SQ-LNS varies to $75 \%, 80 \%$, and $85 \%$. The treatment coverage of wasted children $(\tau)$ was varied between $20 \%$ to $100 \%$, and the vaccination coverage of wasted children was varied between $67.5 \%$ to $100 \%$. 